\title{
Signals Conveyed in the Pulvinar Pathway from Superior Colliculus to Cortical Area MT
}

\author{
Rebecca A. Berman and Robert H. Wurtz \\ Laboratory of Sensorimotor Research, National Eye Institute, National Institutes of Health, Bethesda, Maryland 20892
}

We previously established a functional pathway extending from the superficial layers of the superior colliculus (SC) through the inferior pulvinar (PI) to cortical area MT in the primate (Macaca mulatta). Here, we characterized the signals that this pathway conveys to cortex by recording from pulvinar neurons that we identified by microstimulation as receiving input from SC and/or projecting to MT. The basic properties of these ascending-path PI neurons resembled those of SC visual neurons. Namely, they had brisk responses to spots of light, inhibitory surrounds, and relatively large receptive fields that increased with eccentricity, as well as minimal presaccadic activity. Beyond these basic properties, there were two salient results regarding the modulatory and motion signals conveyed by this ascending pathway. First, the PI neurons appeared to convey only a subset of the modulations found in the SC: they exhibited saccadic suppression, the inhibition of activity at the time of the saccade, but did not clearly show the attentional enhancement of the visual response seen in SC. Second, directional selectivity was minimal in PI neurons belonging to the ascending path but was significantly more prominent in PI neurons receiving input from MT. This finding casts doubt on earlier assumptions that PI provides directionally selective signals to MT and instead suggests that PI derives its selectivity from MT. The identification of this pathway and its transmitted activity establishes the first functional pathway from brainstem to cortex through pulvinar and makes it possible to examine its contribution to cortical visual processing, perception, and action.

\section{Introduction}

A pathway from the superficial layers of the superior colliculus (SC) through the pulvinar to visual cortex has long been considered a second visual pathway to cortex. The functional role of this pathway, and even its existence in the primate, have nonetheless remained in question. We recently identified a pathway through pulvinar from the superficial visual layers of the SC to the visual motion area of cortex, the middle temporal area, MT (Berman and Wurtz, 2010). Using microstimulation, we localized pulvinar neurons that received input from SC, sent output to MT, or did both (relay neurons), and found that they were centered in the inferior pulvinar (PI), particularly within and adjacent to the medial subdivision (PIm) known to project densely to MT (see Fig. 1). In this report, we determine what this pathway conveys to cortex, focusing on two major questions.

The first major question centers on the role of this pathway in transmitting modulatory signals from SC superficial layers to MT. Neurons in the superficial SC show two kinds of modulation related to saccadic eye movements. One is saccadic suppression, the reduction of activity at or around the time of saccade initiation (Goldberg and Wurtz, 1972a; Robinson and Wurtz, 1976).

Received Sept. 9, 2010; revised 0ct. 7, 2010; accepted 0ct. 20, 2010.

This work was supported by the National Eye Institute Intramural Research Program at the National Institutes of Health. We are grateful to Drs. David Leopold, David McMahon, and Michael Schmid for helpful discussions and comments on this manuscript, and to Mitchell Smith, Altah Nichols, Tom Ruffner, and John McClurkin for technical support.

Correspondence should be addressed to Rebecca A. Berman, Laboratory of Sensorimotor Research, National Eye Institute, Building 49, Room 2A50, 49 Convent Drive, Bethesda, MD 20892-4435. E-mail: bermanr@nei.nih.gov. DOI:10.1523/JNEUROSCI.4738-10.2011

Copyright $\odot 2011$ the authors $\quad 0270-6474 / 11 / 310373-12 \$ 15.00 / 0$
This suppression in SC might contribute to the modulation of visual motion responses in MT (Thiele et al., 2002; Ibbotson et al., 2007; Bremmer et al., 2009) via the pathway through PI. The other modulation observed in SC is an enhanced visual response to a stimulus when that stimulus is the target for the next saccade (Goldberg and Wurtz, 1972b). This enhancement might contribute to the increased visual responses observed with shifts of spatial attention in multiple cortical regions (Reynolds and Chelazzi, 2004), including MT (Treue and Maunsell, 1999). The critical question we sought to address is whether these two modulations - saccadic suppression and visual response enhancementare evident in the PI path ascending from SC to MT.

The second question focuses on the visual motion information conveyed by the pulvinar pathway to cortex. We address this question separately because of its relevance to the phenomenon of blindsight (Weiskrantz et al., 1974), in which blind patients with V1 damage can nevertheless respond to certain visual stimulation. One aspect of this residual vision is motion detection (Riddoch, 1917; Zeki and Ffytche, 1998), which likely depends on directionally selective activity in MT (Maunsell and Van Essen, 1983; Britten et al., 1992). MT neurons continue to show residual visual and directional responses after inactivation or ablation of V1 (Rodman et al., 1989; Girard et al., 1992), but additional ablation of SC abolishes these responses (Rodman et al., 1990). Although the prominence of motion detection in blindsight may have been overemphasized (Cowey, 2004), the SC-pulvinar pathway has continued to generate interest as a potential source of motion inputs to MT, particularly given the directional selectivity found in some pulvinar neurons (Petersen et al., 1985). Here, we asked whether PI neurons in the ascending 
path from SC are likely to provide directionally selective signals to area MT.

\section{Materials and Methods}

Experiments were conducted in three male macaque monkeys used in our previous study to identify connected neurons in the pulvinar path from SC to MT (Berman and Wurtz, 2010). The monkeys each had scleral search coils for measuring eye position, and an acrylic base that accommodated both a post for immobilizing the head during experiments and recording chambers. All procedures were approved by the Institute Animal Care and Use Committee and complied with United States Public Health Service policy on the humane care and use of laboratory animals.

\section{Overview of recording and}

\section{microstimulation}

Details of our recording and stimulation procedures are given in our previous paper (Berman and Wurtz, 2010). Briefly, we recorded from single neurons in the pulvinar and used microstimulation to identify their connectivity to the superficial (visual) layers of SC and/or the cortical motion area MT (Fig. 1). In daily experiments, we lowered a recording electrode into the pulvinar and searched for neurons in the inferior and lateral subdivisions. Once we isolated a single neuron, we passed brief pulses of biphasic current through semichronic stimulating electrodes in the SC and MT and looked for evoked spikes in the pulvinar neuron (Lemon, 1984). PI neurons were typically activated from either SC or MT using stimulation currents of $600 \mu \mathrm{A}$ (for additional details, see Berman and Wurtz, 2010). If stimulation activated the neuron through the synapse (orthodromic activation), the pulvinar neuron received input from the stimulated structure. By contrast, if stimulation backfired the neuron through its axon (antidromic activation), the pulvinar neuron projected to the stimulated structure. It is worth noting that, although successful orthodromic/antidromic activation provides clear evidence of connectivity, the failure to observe activation cannot be interpreted as evidence that the neuron lacks a connection to the stimulated structure. A more complete description of our stimulation parameters, including discussion of the strengths and limitations of this technique, is provided in our previous paper (Berman and Wurtz, 2010).

After we established the connectivity of the neuron, we recorded its functional activity while the monkey performed a series of behavioral tasks. We consider the activity of four types of connected pulvinar neurons. We focus primarily on the three types that we classified as belonging only to the ascending pathway: (1) SC-MT relay neurons, which both received SC input and projected to MT, along with neurons that had only one of these identified connections, i.e., (2) those that received input from SC and (3) those that projected to MT. We also briefly describe the activity of a fourth type, pulvinar neurons that received input from MT, which we classified as belonging to the descending pathway from MT to pulvinar. These MT-recipient neurons were mostly independent from the identified ascending-path sample, and there was no overlap between the two samples in our analysis. From our MT stimulation sites (Berman and Wurtz, 2010), we identified no single pulvinar neurons that both received input from MT and projected to MT. However, two of the MT-input neurons also received input from SC. These two neurons were not included in the ascending-path sample but were included as neurons with identified input from MT for the analysis of directional tuning.

\section{Behavioral tasks}

Visual stimuli were backprojected onto a tangent screen located $57 \mathrm{~cm}$ in front of the monkey. For nearly all the neuronal data reported here, stimuli were generated by a DPI projector. For a small number of neu- rons, we also presented laser spot stimuli that were positioned by mirror galvanometers. A computer running REX (Hays et al., 1982) controlled stimulus presentation, reward administration, the recording of eye movements and neuronal activity, and the online display of results. We collected neuronal data during three basic tasks: passive fixation, visually-guided delayed saccades, and memory-guided saccades. A subset of neurons were also tested with a modified fixation task for rapidly mapping receptive field (RF) structure. We report on a variable number of neurons for each task because many cells were lost after only partial data collection, and others did not meet offline criteria for a given analysis.

The fixation task (Fig. $2 \mathrm{~A}$ ) was used to map visual receptive fields, to compare visual responsivity under passive (fixation) versus active (saccade) conditions, and to assess additional properties such as directional tuning. In this task, the monkey began each trial by fixating a small central spot $\left(0.4^{\circ}\right)$ for $100-500 \mathrm{~ms}$. A visual stimulus then appeared for $500-1000 \mathrm{~ms}$ and the fixation spot remained on for an additional $250 \mathrm{~ms}$ before the trial ended. The monkey received liquid reward for maintaining fixation within a $\pm 2^{\circ}$ electronic window throughout the trial. To assess basic visual responsivity, we used a stationary spot stimulus. To assess visual responses to directional motion, we presented a moving stimulus, randomly selected from one of eight canonical directions separated by $45^{\circ}$. For the neurons we report on, we typically used a randomdot pattern $(86 \%)$, with a small portion of neurons tested either using a moving sine wave grating $(13 \%)$ or oriented bar $(1 \%)$.

The visually guided saccade task (Fig. $2 B$ ) was used to assess visual and presaccadic activity and was of primary interest as a measure of visual responsivity compared with the fixation task. Trials began with an initial fixation period of 100-500 ms. A target was then presented in the periphery, but the monkey had to continue fixating centrally for an additional $500-1000 \mathrm{~ms}$ until the fixation spot disappeared, cueing the monkey to make a saccade to the target, which remained illuminated. Reward was given if the monkey attained the target within $500 \mathrm{~ms}$ of fixation offset and maintained gaze at the target for an additional $500 \mathrm{~ms}$. We conducted saccade testing in a subset of neurons using laser spots presented with mirror galvanometers (28\%) and the majority using spots presented with the projector. The target window size was scaled with eccentricity, and ranged on average from $\pm 2^{\circ}$ parafoveally to $\pm 6^{\circ}$ for large saccades $\left(>25^{\circ}\right)$ into the periphery.

The memory-guided saccade task (Fig. 2C) was used to assess activity in relation to eye movements, as it allowed for a separation of visual and 


\section{A Fixation task}

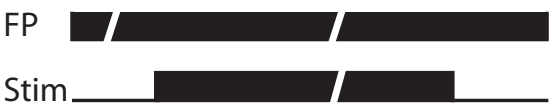

Eye

\section{B Visually-guided saccade task}

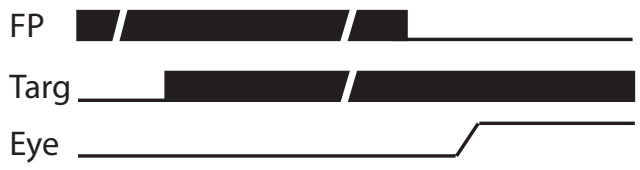

C Memory-guided saccade task

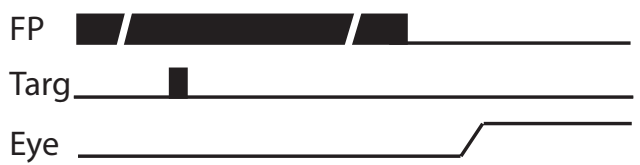

D Rapid mapping task

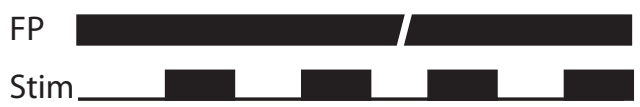

Eye $\overline{\overline{100 m s}}$

Figure 2. Sequence of events for each of the behavioral tasks, described in Materials and Methods. In each panel, the lines represent the appearance and disappearance of the fixation point (FP), the stimulus (Stim), and the expected eye position (Eye). The diagonal openings in some lines indicate variable event timings.

saccade-related events (Hikosaka and Wurtz, 1983). In this task, trials also began with a $100-500 \mathrm{~ms}$ period of initial fixation. A visual target then appeared briefly in the periphery. The target was on for either 50 or $300 \mathrm{~ms}$ (this difference in duration was irrelevant for analysis of saccaderelated activity, which focused on a later part of the trial). After target disappearance, the monkey continued to maintain central fixation for a delay of 500-1000 ms. The central fixation spot was then extinguished, which cued the monkey to make a saccade to the memorized target location. Monkeys received reward for reaching the target within $500 \mathrm{~ms}$ and maintaining fixation there for another $500 \mathrm{~ms}$. During some experiments, the target was reilluminated after successful completion of the memory-guided saccade to provide the monkey with visual feedback to maintain accurate saccades. Data from this visual-feedback epoch were not included in any analysis. The target window size was larger than in the visually-guided task to accommodate increased error of the memoryguided responses and ranged from $\pm 2^{\circ}$ for parafoveal targets to $\pm 10^{\circ}$ for those in the far periphery.

The rapid mapping task (Fig. 2D) was used in a subset of neurons to map receptive field size and structure by presenting multiple spot stimuli in a single trial. In this task, each trial began with an initial fixation period of $250 \mathrm{~ms}$. Subsequently, a set of randomized stimuli appeared for $200 \mathrm{~ms}$ each, separated by a $200 \mathrm{~ms}$ interstimulus interval. The monkey was rewarded for maintaining fixation throughout the presentation of stimuli. Trials typically consisted of eight stimulus presentations. To map the size of the excitatory visual response field, the stimuli varied in location and were randomly sampled from a total of 25 locations $(5 \times 5$ grid $)$ centered at the estimated receptive field center. The diameter of the spot stimulus was held constant and was determined by the distance between grid locations. To measure surround inhibition, the stimuli appeared at a single location, the receptive field center, but varied in size. We typically tested five sizes for a given neuron (e.g., 2, 4, 8, 16, 32 ${ }^{\circ}$ ). The specific sizes varied across neurons depending on the eccentricity and estimated di- ameter of the RF, and ranged overall from $0.5^{\circ}$ (smallest stimulus, tested for a restricted parafoveal RF) to $80^{\circ}$ (largest stimulus, tested for a peripheral $\mathrm{RF}$ ).

\section{Analysis of neuronal activity}

Visual and saccade-related activity. For the analysis of PI visual and saccade-related activity, we analyzed data only from correct trials, usually from 20 trials but from a minimum of 8 trials. We measured visual activity as the average firing rate in a $50 \mathrm{~ms}$ window that began at the onset of the visual response for each neuron. Onset times were determined as the time at which activity exceeded average activity in a $100 \mathrm{~ms}$ prestimulus window (starting $100 \mathrm{~ms}$ before stimulus appearance) by 2 standard deviations for at least $5 \mathrm{~ms}$. We visually verified all onset times. If a neuron had no detectable visual onset to a given stimulus, then we measured activity in a $50 \mathrm{~ms}$ window starting at the median onset time for neurons obtained for the same task in that monkey. We used a paired $t$ test to determine the significance of visual activity compared with baseline activity measured in the $100 \mathrm{~ms}$ before stimulus onset.

We assessed attentional modulation of visual activity by comparing the average response to the same stimulus in the visually-guided saccade task and the passive fixation task. As described in the preceding paragraph, we measured activity in the $50 \mathrm{~ms}$ window beginning at the response onset of the neuron. Onset times were determined independently for fixation and saccade tasks to avoid any possible bias, and we found no significant difference in these times between the two tasks (Wilcoxon's signed rank, $p>0.8$ ).

We measured presaccadic activity in a $50 \mathrm{~ms}$ window that began $50 \mathrm{~ms}$ before the saccade start, which was taken as the time at which eye velocity exceeded $50 \%$. We measured postsaccadic activity in a $50 \mathrm{~ms}$ window that began $70 \mathrm{~ms}$ after the beginning of the saccade (Robinson and Wurtz, 1976). This postsaccadic window preceded the time of target reappearance on trials in which the monkey received visual feedback, ensuring that the measure of postsaccadic activity was uncontaminated by visual stimulation. We considered changes in either presaccadic or postsaccadic activity to be significant by comparing them (paired $t$ test) to ongoing activity, which we measured in a $100 \mathrm{~ms}$ presaccadic baseline epoch that began $200 \mathrm{~ms}$ before saccade start. We used this baseline window even for the postsaccadic comparison because we knew that a few cells had significant increases in activity just before the saccade, which would have falsely exaggerated the postsaccadic suppression of activity. We conducted an additional analysis of saccadic suppression to determine its start and duration in a subset of neurons. The start of suppression was taken as the time at which activity (represented as a spike density function with $8 \mathrm{~ms}$ Gaussian smoothing) fell below a threshold of $25 \%$ of average activity in the presaccadic baseline window and remained below this threshold for at least $15 \mathrm{~ms}$. The end of suppression was the time at which the firing rate exceeded this threshold and remained above it for at least $15 \mathrm{~ms}$. We searched for the start of suppression in a window -100 to +150 ms relative to saccade onset and searched for the end of suppression up to $300 \mathrm{~ms}$ after saccade onset (Robinson and Wurtz, 1976; Ibbotson et al., 2008; Bremmer et al., 2009; Hafed and Krauzlis, 2010). We visually verified the start and duration values for each neuron.

Receptive field structure. Although the demands of the experiment did not afford time to conduct a detailed study of the RF structure of identified PI neurons, we obtained estimates of two parameters: size and surround inhibition. To estimate RF size, we measured the visual responses for stimuli presented at a variety of locations. We first obtained this estimate manually by varying stimulus position in the fixation task and qualitatively determining the horizontal and vertical extent of the RF. In a subset of neurons, we also used the automated rapid mapping task to quantify activity at 25 grid locations. For each location, we computed average visual activity in a $50 \mathrm{~ms}$ window that began with the median neuronal onset time, which was determined by searching for the start of the visual response (as described previously) for each location and taking the median of the detected times. We visually inspected timing data for each cell to ensure that the response was captured for all locations. We analyzed all neurons for which there was a detectable visual response at one or more locations. Average visual activity was compared with that of a baseline epoch of $100 \mathrm{~ms}$ that began $50 \mathrm{~ms}$ before stimulus onset. We 
measured the horizontal and vertical diameter of the RF by determining the locations at which this visual response was significantly greater than baseline activity ( $p<0.05$, paired $t$ test). We took the average of the horizontal and vertical diameters as the estimate of RF size (diameter). For neurons in which we estimated size with both automated and manual methods, we found close agreement between the two and therefore include the data obtained using only the manual estimate. To measure the visual surround inhibition, we presented spots with increasing diameters in the center of the RF. We used either the rapid mapping task, in which multiple diameters were presented on a single trial, or the basic fixation task, in which only one diameter was presented on a single trial. We measured average visual activity in a $50 \mathrm{~ms}$ window starting at the median neuronal onset time across diameters, determined using the same approach described for locations. From this, we took the small spot size that produced the best response as the optimal stimulus size (often the smallest stimulus did not drive the neuron most strongly). The large spot size was the biggest size tested and, in order for the neuron to be included in analysis, its size had to exceed the estimated RF diameter. We then determined the presence of an inhibitory surround by computing a surround inhibition index, large/small, which was the average response to the large stimulus divided by the response to the optimal small stimulus. For the analyzed sample, the median large stimulus was $20^{\circ}$ in diameter and the median optimal small stimulus was $4^{\circ}$.

Directional tuning. We measured responses to moving stimuli in a 100 $\mathrm{ms}$ window starting at the neuronal onset time, which was set to the median of detectable onset times for all eight directions. We computed a directionality index (DI) for each neuron, using the following formula: $1-(n / p)$, where $p$ is the firing rate for the preferred direction minus spontaneous activity, and $n$ is the firing rate for the null direction minus spontaneous activity (Maunsell and Van Essen, 1983). The preferred direction was the direction that elicited the highest average firing rate in the response window, and the null direction was $180^{\circ}$ opposite from preferred. We measured spontaneous activity as the grand average of activity from all eight directions, computed in a $100 \mathrm{~ms}$ baseline window before the appearance of the motion stimulus. DI values near zero indicate that the neuron responded similarly for preferred and null directions (i.e., no tuning), and those near 1 indicate strong directional selectivity. Values $>1$ indicate that the activity of the neuron in the null direction was suppressed below the spontaneous rate. Note that this measure of directionality does not identify bidirectional tuning, in which the neuron has a preference along an axis. For inclusion in the analysis of directional tuning, the neuron had to have a significant visual response at least for the preferred direction compared with its baseline activity (paired $t$ test). The neuron also had to have at least 64 trials total from all eight directions, with at least seven trials per direction.

\section{Results}

\section{Location and connectional identity of recorded pulvinar neurons}

To understand the functional signals conveyed by the pulvinar path from SC to MT, we recorded the activity of pulvinar neurons that were physiologically identified as having connections to the superficial layers of SC and/or area MT. We identified a total of 313 pulvinar neurons that were either antidromically or orthodromically activated by microstimulation of SC and/or MT (Berman and Wurtz, 2010). Consistent with recent anatomical findings (Lyon et al., 2010), these neurons were located almost exclusively in PI and were clustered in two zones, one adjacent to the medial geniculate nucleus (MGN) and the other adjacent to the lateral geniculate nucleus (LGN). The MGN-adjacent zone comprised the more dense concentration of identified neurons and included two subdivisions that were histologically verifiedPIm (known to have strong projections to MT) and PIp (the medial-most subdivision). The LGN-adjacent zone, where we found evidence for a smaller cluster of connected neurons, likely corresponds to the lateral shell region of PI (Cusick et al., 1993; Lyon et al., 2010). We found no consistent location-dependent differences among the identified neurons, and will refer to all of them as PI neurons. Of these, we were able to record functional data from 116 neurons while the monkeys performed behavioral tasks. Our primary focus is on the PI neurons belonging to the ascending tecto-cortical pathway (Fig. 1, black arrows; $n=99$ ), which comprised three classes: those that received input from SC $(n=58)$, sent output to area MT $(n=28)$, or had both SC input and MT output (SC-MT relay neurons; $n=13$ ).

We first describe the basic visual and saccade-related activities of these ascending-path neurons, and then concentrate on addressing two questions about the role of the pulvinar path from SC to MT: (1) Do neurons in this ascending PI pathway express the modulations of activity observed in SC? (2) Do neurons in this ascending PI pathway have visual motion responses consistent with conveying directionally selective information to MT? In answering this second question, we also report on a fourth class of PI neurons, those that received input from area MT and, as such, belonged to the descending path from cortex to pulvinar (Fig. 1, white arrow; $n=17$ ).

\section{Visual and saccadic activity in the ascending pathway \\ Responses to spots}

An obvious starting point is to ask whether neurons in the ascending pulvinar path through PI have the signature visual responses observed in the superficial layers of the SC. We found that nearly all SC-MT relay neurons in our sample (12 of 13) were strongly visual, and had receptive fields wherein visual stimuli elicited a phasic burst of action potentials after stimulus onset in the fixation task. The activity of an example relay neuron (Fig. $3 A$ ) shows that it fired strongly when a small spot of light was 
A Example PI receptive field mapping

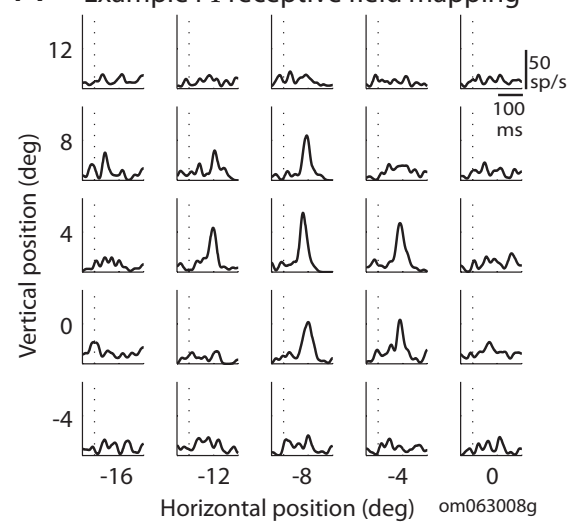

C Example PI surround inhibition

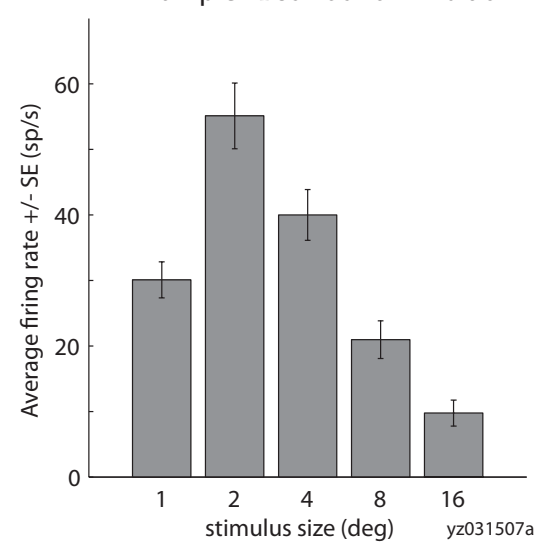

B

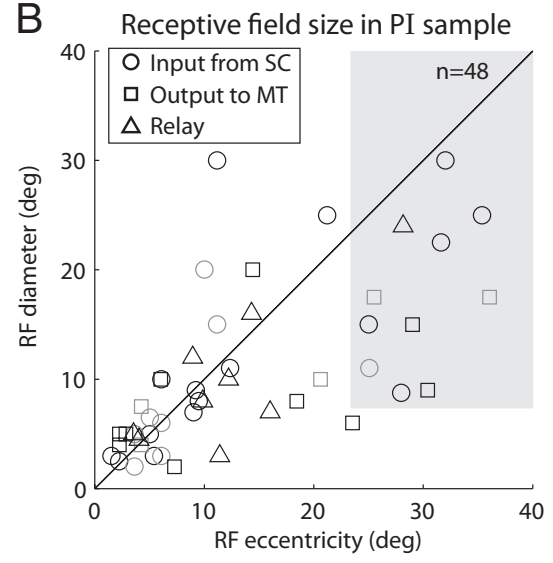

D Surround inhibition in PI sample

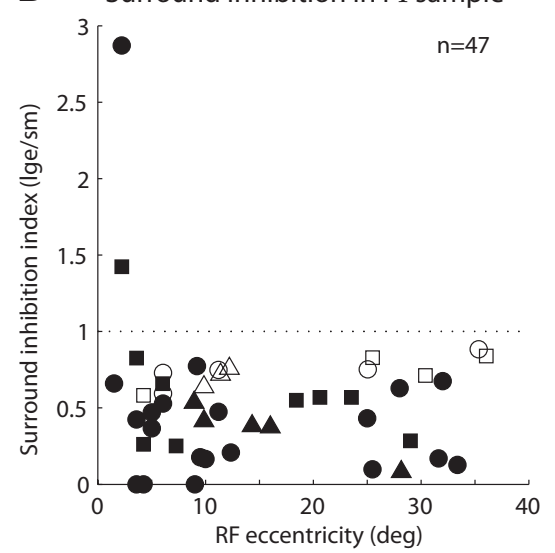

Figure 4. Receptive field structure of PI neurons in the ascending path. $A$, RF mapping for an example relay neuron. Each panel shows the spike density function of the visual responses for one of 25 locations in a $5 \times 5$ grid. Data are aligned on stimulus onset (dotted vertical line). $\boldsymbol{B}$, Relationship between RF eccentricity ( $x$-axis) and RF diameter ( $y$-axis) in the sample of PI neurons. Symbol color indicates the mapping method (black, automated; gray, manual) and the shaded region indicates large-eccentricity fields that could not be fully mapped. C, Surround inhibition in an example neuron that received input from SC. Bars show the average response ( $\pm S E ; y$-axis) to visual stimuli of varying diameter ( $x$-axis). $\boldsymbol{D}$, Surround inhibition in the sample of neurons. Graph shows the surround inhibition index (response to largest stimulus size/response to optimal small stimulus, $y$-axis) as a function of RF eccentricity ( $x$-axis).

presented in its receptive field. Visual responses were likewise robust for the other classes of neurons in the ascending path, i.e., those for which we could identify only input from SC or only output to MT. Figure $3 B$ plots the average visual activity against baseline activity for each ascending-path neuron tested in the fixation task. Average visual activity was significantly greater than baseline activity in most individual neurons ( 63 of 79; $80 \%$; filled symbols; $p<0.05, t$ test), and for the sample ( $p<0.0001$, Wilcoxon's signed rank). Another feature of neurons in the superficial SC is that they respond well to stationary spots of light and rarely require an oriented or directional stimulus (Goldberg and Wurtz, 1972a). The fact that we observed significant visual responses in $80 \%$ of this sample of PI neurons, all tested with stationary spots, shows that the majority did not require an oriented or directional stimulus.

There were no significant differences in the strength of visual activity among the three types of ascending-path neurons- those identified with only input from SC, only output to MT, or SC-MT relay neurons (all comparisons $p>0.1$, Wilcoxon's rank sum). We likewise found that activity profiles did not differ significantly for these three neuron classes in subsequent analyses and therefore combine them into a single group.

\section{Receptive field structure}

All PI neurons in the ascending SC-MT pathway had RFs contralateral to the recorded hemisphere. The average eccentricity of the RF centers was $12.4^{\circ}$ (range, $1.1-36.1^{\circ}$ ) and average elevation was $36.9^{\circ}$. We successfully obtained more detailed information about RF structure in about one-half of the ascending-path neurons. To express RF size, we simply took the average of the horizontal and vertical extent as the estimate of diameter. The RF of an example relay neuron (Fig. $4 A$ ), which gave a robust response in the automated rapid mapping task, was centered at $9^{\circ}$ eccentricity and had an estimated diameter of $8^{\circ}$. For the sample of PI neurons, we found that the size of the RF tended to increase with eccentricity (Fig. $4 B$ ). We were unable to determine the full extent of RFs for neurons at larger eccentricities (shaded region) because their borders were beyond the stimulus presentation screen. For neurons with eccentricities of $<25^{\circ}$, where screen size was not a limiting factor, RF size was approximately equal to RF eccentricity. For these neurons, the median ratio of diameter/eccentricity was 1.11 (range, 0.25-2.6; $n=37$ ).

We next investigated a second property of RF structure, surround inhibition, which has been observed both in superficial SC and in area MT (Goldberg and Wurtz, 1972a; Allman et al., 1985). This phenomenon is also called "surround suppression," but we use the term "inhibition" in this report to minimize confusion with the separate phenomenon of saccadic suppression. We tested for surround inhibition in PI neurons by presenting spot stimuli of randomly varying size during the fixation task. Sizes differed across neurons depending on RF diameter and eccentricity, but for each neuron, sizes ranged from small stimuli presented well within the boundaries of the receptive field to large stimuli that exceeded these boundaries. We computed a simple index to summarize the surround inhibition, large/small, where large is the average activity in response to the largest stimulus presented to the neuron (at minimum, this stimulus had to exceed the estimated RF diameter) and small is the average activity in response to the optimal small stimulus (see Materials and Methods). Values $<1$ indicate that the neuron fired less to the large stimulus and thus exhibited surround inhibition. The example neuron in Figure $4 C$ had strong surround inhibition. It responded best to $2^{\circ}$ spots (its optimal small stimulus) but successively larger stimuli evoked smaller responses, and its activity was greatly diminished for the largest stimulus $\left(16^{\circ}\right)$. The surround inhibition index for this neuron was 0.18 , indicating a $\sim 80 \%$ decrease in its response. The population data in Figure $4 D$ show that most ascending-path neurons had index values $<1$, regardless of their receptive field eccentricity. Furthermore, the majority of individual neurons $(68 \%)$ had a significantly inhibited response to the largest stim- 
ulus compared with the optimal small stimulus (filled symbols; $p<0.05, t$ test). The overall picture that emerges is that PI neurons in the ascending path from SC to MT convey the basic visual responses observed in the superficial SC.

\section{Weak presaccadic activity}

In contrast to their robust visual responses, we expected that ascending-path neurons would not have substantial saccaderelated activity for two reasons. First, as described previously, our SC stimulating electrodes targeted the superficial layers where neurons have prominent visual activity but no presaccadic bursts as seen in the deeper layers of SC. Second, previous studies have reported little to no presaccadic activity in the general population of neurons in the pulvinar (Petersen et al., 1985; Robinson et al., 1986). In keeping with this expectation, we found minimal presaccadic activity in the PI neurons identified as receiving input from SC and/or projecting to MT. An example neuron with input from SC (Fig. 5A), recorded during the memory-guided saccade task, exemplifies the pattern we encountered most commonly: robust visual activity but an absence of presaccadic activity. We nonetheless observed presaccadic activity in a handful of PI neurons, including a relay neuron (Fig. $5 B$ ) that had the strongest presaccadic activity in the ascending-path sample. Even for this PI neuron, however, the presaccadic activity was small compared with its visual response and did not resemble the robust bursts of intermediate SC. Figure $5 C$ shows the presaccadic activity of all PI neurons recorded in the memory-guided saccade task. Average presaccadic activity for this sample did not differ significantly from activity in the baseline epoch ( $p=0.8$, Wilcoxon's signed rank). Of these 75 neurons, only six (8\%) had a significant increase in firing before the eye movement and one had a significant decrease.

All six of the PI neurons with significant presaccadic activity had identified input from SC (two were relay neurons), which led us to ask whether their presaccadic activity reflected activation from the intermediate, saccade-related layers of the SC. Two circumstances could cause such activation. First, the stimulating electrode in SC could have been located more deeply. In this case, we would expect to find very low current thresholds for evoking saccades with a train of pulses at the SC stimulation site. For PI neurons with SC input, however, greater presaccadic activity was not associated with lower saccade thresholds at the SC site (regression, $r^{2}=0.004 ; p>0.6$ ). A second circumstance-the use of high currents for the single pulses that activated the PI neuroncould also involve the intermediate layers. Here, too, there was no relationship between the magnitude of PI presaccadic firing and the current threshold for activating the PI neuron from SC $\left(r^{2}=\right.$ $0.020 ; p>0.2)$. Furthermore, the six PI neurons with significant presaccadic activity had saccade and activation thresholds that were consistent with stimulating the superficial layers. Thus, the weak but significant presaccadic activity seen in a small number of PI neurons is unlikely to arise from inadvertent activation of the intermediate SC.

To summarize the basic properties of the majority of PI neurons in the ascending path: they had strong visual responses to spots of light, RFs that scaled with eccentricity, and RFs with inhibitory surrounds. Presaccadic responses were weak but occasionally significant. These findings demonstrate that the basic visual and presaccadic signals in this PI relay of the collicular-cortical pathway correspond closely to those of the superficial SC.
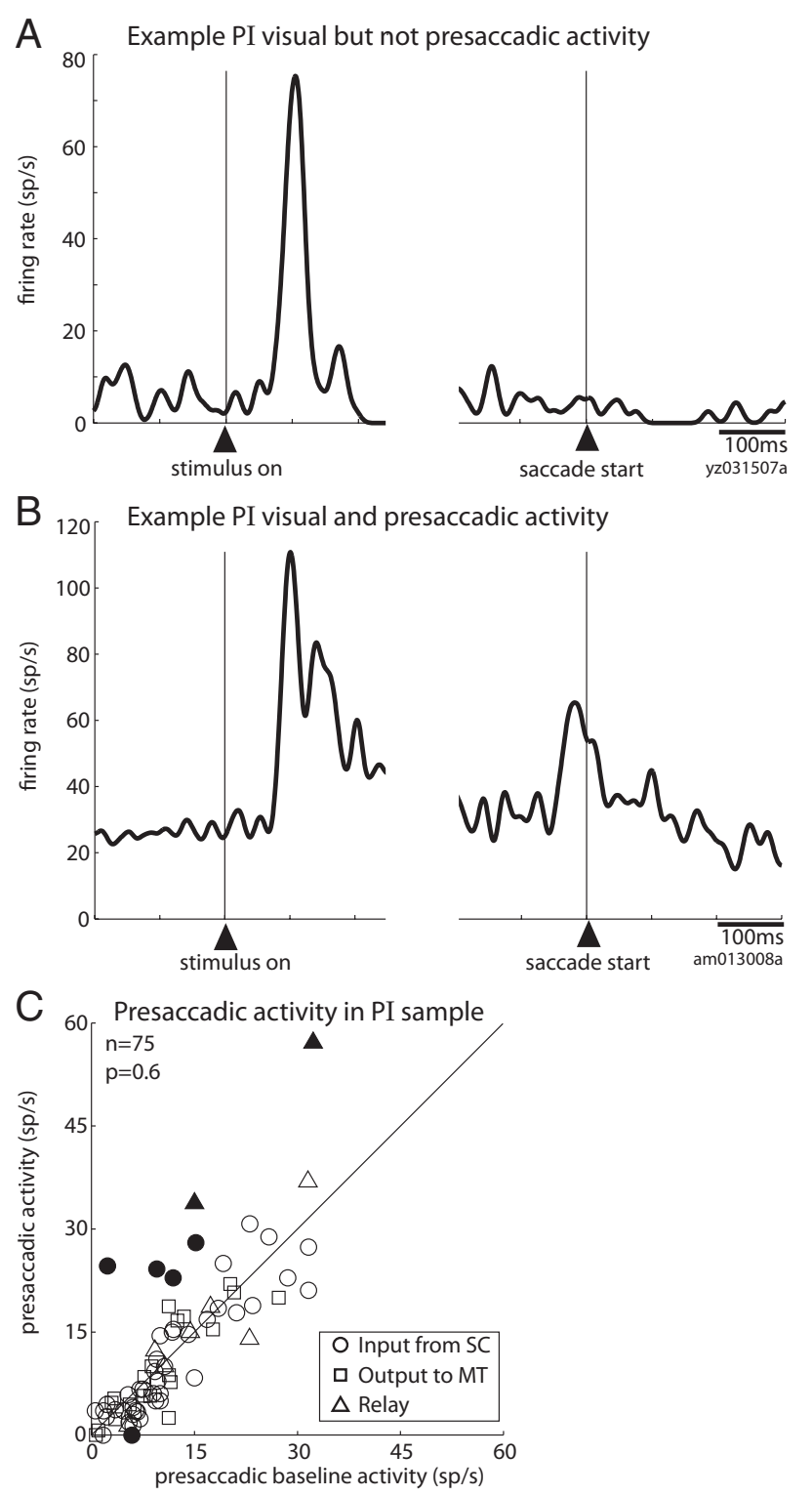

Figure 5. Presaccadic activity is unusual in pulvinar neurons of the ascending path. A, Activity of a representative neuron with input from $\mathrm{SC}$ that has a visual response but no presaccadic burst. Data from the memory-guided saccade task are aligned on the onset of the visual stimulus (left panel) and on the start of the saccade (right panel). B, Activity of an example relay neuron with the strongest presaccadic activity in our sample. $C$, Average presaccadic activity in the memory-guided saccade task is plotted against ongoing presaccadic baseline activity for each neuron in the ascending path. Conventions are as in Figure $3 B$.

\section{Does the pulvinar transmit modulations from SC?}

Saccadic suppression

A striking feature of activity in the superficial SC is its modulation by eye movements. Each time the eyes move, the visual scene sweeps across the retina, yet this visual stimulation fails to activate many of the visually responsive neurons in the superficial SC (Goldberg and Wurtz, 1972a). In many SC neurons, saccadic suppression is also observed as an inhibition of ongoing activity just after a saccade, at the time when any visual response caused by the saccade is most likely to occur (Robinson and Wurtz, 1976). Previous experiments demonstrated that the suppression results from a corollary discharge (a copy of the eye movement command) to these superficial layer SC neurons (Richmond and Wurtz, 1977). Comparable saccadic suppression has been found 
A Example PI saccadic suppression

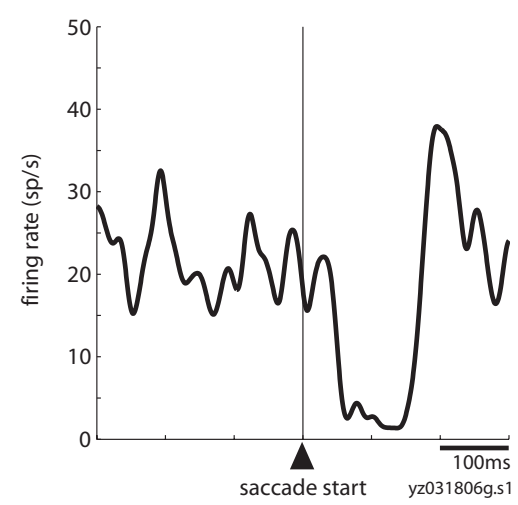

B Saccadic suppression in PI sample

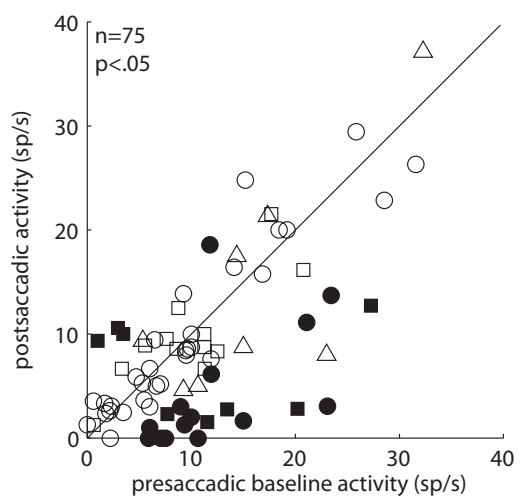

Figure 6. The ongoing activity of some ascending-path PI neurons is suppressed after saccades. $A$, Saccadic suppression of a representative neuron that projects to area MT. Data from the memory-guided saccade task are aligned on the beginning of the saccade. $\boldsymbol{B}$, Average postsaccadic activity is plotted against presaccadic baseline activity for each neuron with SC input and/or MT output. Conventions are as in Figure $3 B$.

in the general population of pulvinar neurons (Robinson et al., 1986, 1991). Here, we asked whether saccadic suppression was present in the PI neurons identified as belonging to the ascending path from SC to MT. We investigated the suppression of ongoing activity using the memory-guided saccade task. In this task, both the fixation point and the target stimulus disappear before the monkey's eye movement, minimizing any effect of visual stimulation on neuronal activity. We found significant saccadic suppression in a subset of ascending-path PI neurons. The saccade-related activity of an example neuron projecting to MT (Fig. 6A) shows a sharp decrease in activity immediately after the eye movement. The PI population scatterplot (Fig. $6 \mathrm{~B}$ ) shows that, although the majority of neurons were not significantly modulated in the postsaccadic epoch, the sample exhibits a significantly lower average activity just after the saccade than well before it $(p<0.05$, Wilcoxon's signed rank). In one-third of PI neurons (22 of 75), postsaccadic activity was significantly different from ongoing activity, and a clear majority of these were significantly suppressed (18 of $22)$. In a small number of PI neurons with suppression $(n=4)$, we were also able to record saccade-related activity using mirror galvanometers to present the visual stimuli. With this system, the monkey's saccades were made in total darkness, ruling out any visual source of the modulation. The saccadic suppression persisted in complete darkness, suggesting that the modulation is of an extraretinal origin.

We conducted an additional analysis to determine the temporal dynamics of saccadic suppression. Recent studies of the time course of saccadic suppression in extrastriate cortex and in the intermediate SC suggest that suppression can begin even before the eyes move (Ibbotson et al., 2008; Bremmer et al., 2009; Hafed and Krauzlis, 2010). These studies note that the neuronal effects may correspond to perceptual measures of suppression (Ross et al., 2001; Wurtz, 2008) and again point toward an internal corollary discharge signal as a source of modulation. We wanted to characterize the timing of suppression of ongoing activity in the PI path and were particularly interested in the possibility that suppression might be detectable in some neurons before saccade initiation. We limited this analysis to the subset of 18 neurons that showed significant suppression, and we found a clear start and duration in 16 of them using a search window beginning 100 ms before saccade onset (see Materials and Methods). Suppres-

sion began as early as $60 \mathrm{~ms}$ before saccade initiation and as late as $138 \mathrm{~ms}$ afterward. In five neurons, suppression began either before or at virtually the same time as (within $10 \mathrm{~ms}$ ) saccade initiation. This early suppression is consistent with example data from superficial SC (Goldberg and Wurtz, 1972a) and suggests the influence of a corollary discharge signal. The remaining 11 neurons had postsaccadic suppression starting at least $41 \mathrm{~ms}$ after saccade initiation. For the sample of 16 neurons, the mean start of suppression was $57 \mathrm{~ms}$ after saccade initiation with a mean duration of $107 \mathrm{~ms}$. We conclude that a subpopulation of PI neurons in the path from SC to MT exhibit perisaccadic suppression, and that these suppression signals occur at a time when they could act on the visual input caused by eye movements.

\section{Enhanced visual responses}

A second feature of the visual responses of superficial SC neurons is that they can be strongly modulated by the behavioral relevance of the visual stimulus. Specifically, if the monkey intends to make a saccade to a stimulus in the RF of an SC neuron, then the visual response evoked by that stimulus is enhanced compared with the response in a passive fixation task (Goldberg and Wurtz, 1972b). Is this enhancement also present in PI neurons that belong to the ascending path from SC to MT? We measured the visual response of the PI neurons during the fixation task and compared it with the response during the visually-guided saccade task, in which the same physical stimulus was now the target of a saccade. On average, ascending-path PI neurons were unmodulated by the behavioral relevance of the stimulus. For example, the visual response of the PI relay neuron in Figure 7A is virtually identical for the two tasks. A portion of individual PI neurons ( 8 of $67 ; 13 \%)$ exhibited a significantly different visual response between the fixation and visually-guided saccade tasks. Interestingly, however, the sign of this attentional modulation was not systematic. Instead, we observed examples in which the visual response was significantly enhanced in the saccade task (Fig. $7 B$ ) as well as examples in which the response was significantly reduced (Fig. $7 C$ ). This variability in the sign of modulation contributed to the absence of any significant attentional effect in the sample of ascending-path PI neurons (Fig. 7D), for which we observed no significant difference between the passive fixation task and the saccade task ( $p=0.6$, Wilcoxon's signed rank).

We investigated two additional points to understand this pattern of results more fully. We first focused on the presence of modulation observed in a portion of PI neurons and asked whether certain properties might predict this modulation. For example, was a particular class of connected PI neurons-those with input from SC, output to MT, or both (relays) - more likely to be affected by behavioral relevance? We found no differences among these three classes in the magnitude of enhancement $(p>$ 0.17 for all comparisons). We were also unable to detect any pattern in the localization of these neurons within the pulvinar that distinguished those with modulation from those without. We further considered the possibility that the magnitude of modulation might be related to the magnitude of the visual response 
of the PI neuron during the fixation task; for example, enhancement might be larger for neurons with a smaller visual response. As is clear from the scatterplot in Figure $7 D$, however, this parameter also failed to predict modulation. There were no obvious properties that differentiated the PI neurons with modulation from those without.

Our results for the PI neurons in the ascending path were thus quite different from the previous results in the SC. We saw enhanced visual responses during the saccade task in $<5 \%$ of PI neurons, compared with $40-50 \%$ previously reported for superficial SC neurons (Goldberg and Wurtz, 1972b; Wurtz and Mohler, 1976). This discrepancy could reflect true differences in the response properties of these two populations, but it could also reflect differences between our current experimental procedures and those of the previous studies. To examine this possibility, we recorded from 33 neurons in superficial SC under the identical experimental conditions used for recording from PI neurons. In our SC sample (Fig. 7E), the visual response clearly increased more than it decreased: $18 \%$ of the neurons had a significant enhancement, such as that in Figure $7 F$, whereas only a single neuron $(3 \%)$ had a significantly reduced response. The percentage of SC enhanced neurons is smaller than in the previous reports (Goldberg and Wurtz, 1972b; Wurtz and Mohler, 1976). This difference may be attributable to two features of the present experiment-the use of higher stimulus luminances, and the use of a fixation task verified by eye position instead of the earlier fixation task, in which the monkey had to detect the dimming of the central fixation point. These two changes could, for example, contribute to higher visual responses in the fixation task and thus create a ceiling effect, making it difficult to detect an enhancement in the saccade task. This particular explanation seems unlikely, given that we observed enhancement even for PI and SC neurons with very robust responses in the fixation task. It is nevertheless a real possibility that these procedural differences reduced the likelihood of observing enhancement in SC in the present study and also contributed to the lower percentages of modulation in the pulvinar. Despite this possibility, however, we found that enhancement was significantly more prominent in superficial SC neurons than in PI neurons under identical experimental conditions. Specifically, we observed enhancement at a significantly higher frequency in SC than in PI ( 6 of 33 vs 3 of 67; Fisher's exact test, $p<$ $0.05)$. These data imply that the enhanced visual responses seen in the superficial SC during the saccade task are not prominently represented in this identified PI pathway.
A Example PI neuron with no modulation

B Example PI neuron with enhancement
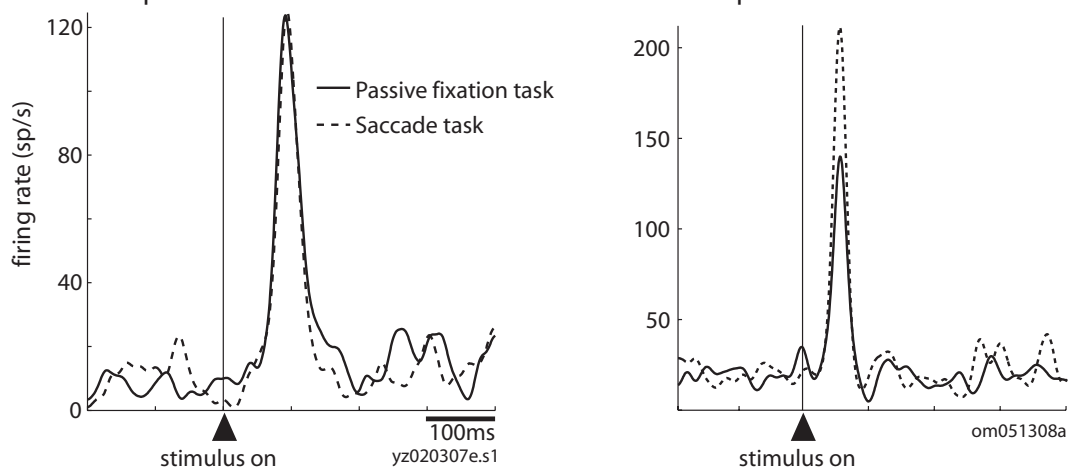

C Example PI neuron with decrease

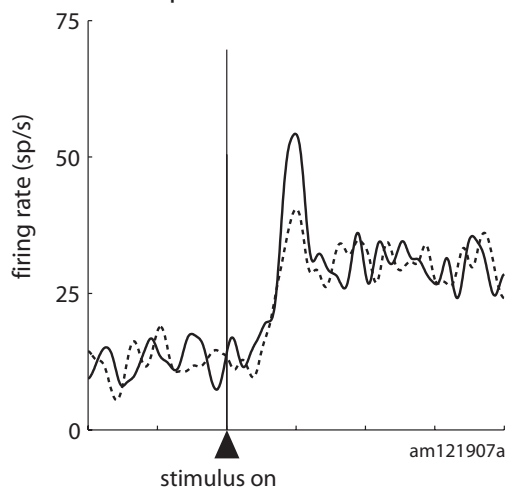

D Visual modulation in PI sample

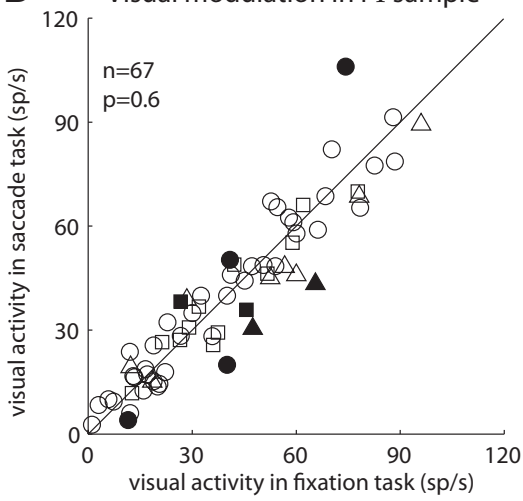

$\mathrm{F}$

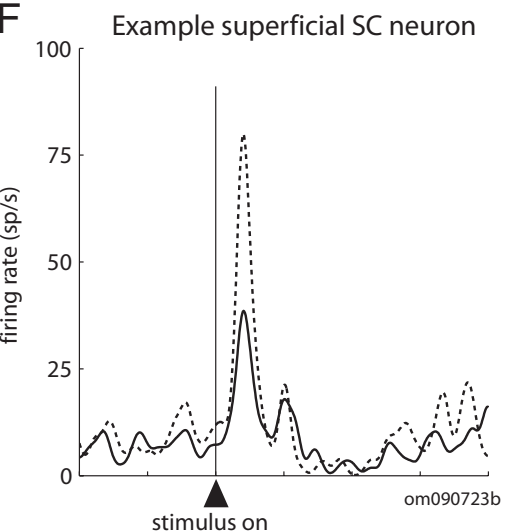

Figure 7. The intention to make a saccade rarely modulates the visual response of PI neurons in the ascending path. $A$, Similar visual response of an example relay neuron to the same physical stimulus during the passive fixation task (solid line) and the visually-guided saccade task (dotted line). $\boldsymbol{B}$, Increased visual response of an example PI neuron that received input from the SC. $\boldsymbol{C}$, Decreased visual response of an example PI neuron that projected to MT. $\boldsymbol{D}$, Average visual responses for the visually-guided saccade task ( $y$-axis) are plotted against those for the fixation task ( $x$-axis) for the sample of ascending-path PI neurons. $E$, Average visual responses for the sample of superficial $S C$ neurons (same conventions as in $\boldsymbol{D}$ ). $\boldsymbol{F}$, Increased visual response of an example superficial SC neuron to the stimulus during the saccade task compared with the fixation task.

\section{Does the ascending pathway convey directional motion information to MT?}

Previous studies have reported that some pulvinar neurons exhibit preferential responses for specific directions of motion (Petersen et al., 1985). Such selectivity has been proposed to contribute to the residual detection of motion observed in blindsight patients. A central question, then, is the degree to which directional tuning is present in the pulvinar neurons that belong to the path ascending from SC to MT. We addressed this question by first assessing the directional selectivity for visual motion of the ascending-path neurons. We determined the visual responses of 
A

Untuned PI relay neuron

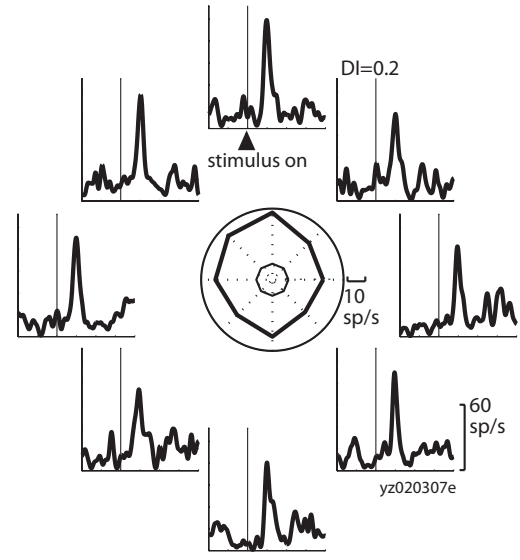

C Tuned PI neuron with input from MT

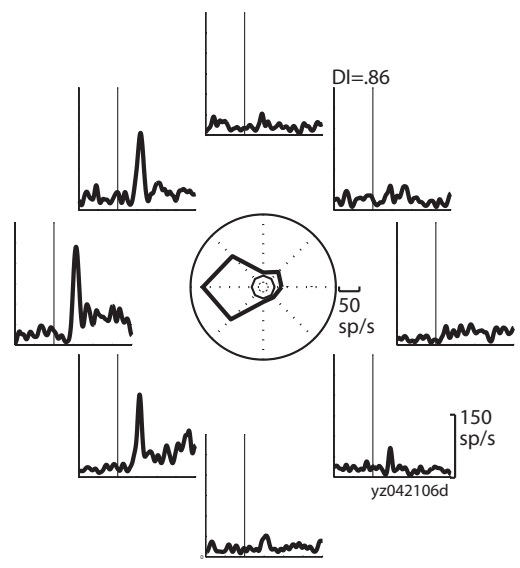

B

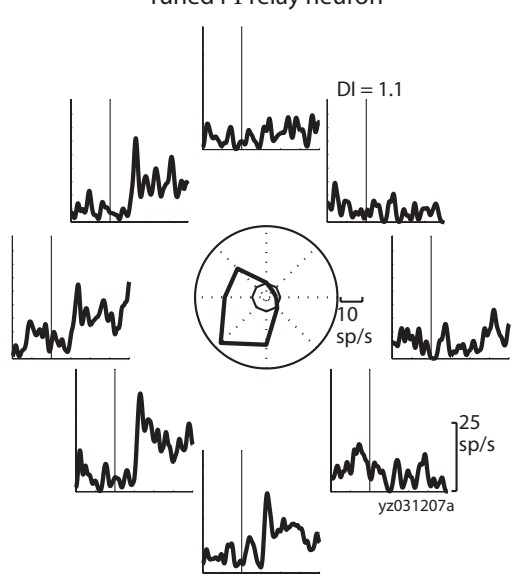

D

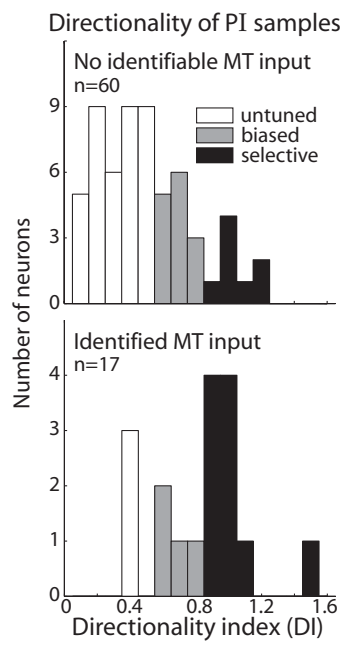

Figure 8. Directional tuning is atypical in PI neurons without identifiable input from area MT. $A$, Responses of an example relay neuron with characteristic absence of directional tuning. Each panel shows the response of the neuron to one of the eight cardinal directions of motion, aligned on the appearance of the motion stimulus. The polar plot in the center summarizes the directional tuning of the neuron: the thick line shows average activity in the directional-response epoch for each direction, and the thin line shows average spontaneous activity across all eight directions, used to compute the DI (see Materials and Methods). $\boldsymbol{B}$, Directional responses of the relay neuron with strongest directional tuning in the sample of ascending-path PI neurons. C, Directional responses of a PI neuron receiving input from MT, exemplifying the directional tuning commonly observed in the sample of MT-input cells. $\boldsymbol{D}$, Distribution of DI values for the sample of ascending-path neurons with no identifiable input from MT (top panel) and the sample of descending-path neurons identified as receiving projections from MT (bottom panel). The number of neurons in a bin is indicated on the $y$-axis, and the DI values for the bins are on the $x$-axis. Shading (legend) indicates the tuning classification.

PI neurons to stimuli moving in eight cardinal directions and then computed a directionality index, DI, for each neuron. The DI was computed using the standard formula $1-(n / p)$, where $p$ is the response to the preferred direction and $n$ is the response to the direction $180^{\circ}$ opposite the preferred, both corrected for spontaneous activity (Maunsell and Van Essen, 1983). On the extremes of this scale, a value of zero indicates identical responses to preferred and null directions, and values $>1$ indicate an inhibitory response to the null direction. Following the convention of Gizzi et al. (1990), we considered DI values between 0.5 and 0.8 to signify a directional bias, and values $>0.8$ to signify directional selectivity.

We found that the PI ascending-path neurons were mostly untuned. For example, the relay neuron in Figure $8 \mathrm{~A}$ responded with a burst of activity regardless of the direction of motion. We nevertheless encountered directional tuning in some ascendingpath neurons, including a relay neuron (Fig. $8 B$ ) that strongly preferred motion down and to the left. This kind of tuning, however, was atypical in the sample of ascending-path PI neurons. We then asked whether directional tuning was more prominent in a separate sample of PI neurons, those that we had identified as receiving input from area MT. [Note that we found no single PI neurons with both input from MT and output to MT from our stimulation sites (Berman and Wurtz, 2010).] The example PI neuron with input from MT (Fig. 8C) illustrates the directional selectivity commonly found in this subset of identified pulvinar neurons. This neuron had a clear preference for stimuli moving to the left.

The key question that emerges is whether directional selectivity is more likely to be present in PI neurons that receive input from MT than in neurons belonging to the ascending path to MT. We divided the PI neurons into two samples, those that had identifiable input from MT and those that did not. Figure $8 D$ shows the distribution of DI values for these two groups of neurons. Directional tuning is clearly more prevalent in PI neurons with identifiable MT input compared with those without. The distribution of DI values was significantly higher for the PI neurons with known input from MT (median, 0.86) than those without (median, $0.41 ; p<0.0001$, Wilcoxon's rank sum). When we classified neurons by standard criteria (Gizzi et al., 1990), we found that the large majority of the PI neurons with MT input were either directionally selective $(10$ of $17 ; 59 \%)$ or directionally biased ( 4 of $17 ; 24 \%$ ). Even among the neurons with DI values $\leq 0.5$, two had bidirectional selectivity. By contrast, of the PI neurons with no identifiable MT input, the majority $(63 \%)$ were untuned. We found that approximately one-fourth of these neurons were directionally biased (14 of 60;23\%) but far fewer were selective ( 8 of $60 ; 13 \%)$. In sum, directional signals are significantly more likely to be found in PI neurons that receive input from MT than in PI neurons in the projecting path from SC to MT.

\section{Discussion}

We previously established a functional pathway from the SC superficial layers through the PI to cortical area MT (Berman and Wurtz, 2010). In the present experiment, we identified several signals that this pathway conveys. The basic response properties of connected neurons in the ascending PI path were similar to those of SC visual neurons. They had a vigorous response to spots of light, indication of an inhibitory surround, and relatively large receptive fields that increased with eccentricity. Presaccadic activity was weak when present, consistent with PI activation from microstimulation of the superficial SC. Our discussion focuses on two central findings: the selective transmission of modulatory 
signals from the SC, and the relative lack of directionally tuned motion responses in the ascending pulvinar path to MT.

\section{Transmission of modulatory visual signals}

Far from being a passive process, visual perception is actively shaped by motor and cognitive signals. Both eye movements and attention modulate neuronal activity, not only in cortex but also in subcortical structures such as the superficial SC. This raises the possibility that certain modulations are transmitted to cortex via subcortical pathways. The identification of the PI pathway from SC to MT provides an opportunity to examine this possibility. We expected that neurons in this pathway would exhibit two kinds of modulation present in the superficial SC.

The first is saccadic suppression, a reduction of visual sensitivity at the time of saccades (Ross et al., 2001; Wurtz, 2008). Saccadic suppression of neuronal activity occurs in the superficial SC (Goldberg and Wurtz, 1972a; Robinson and Wurtz, 1976) and is likely attributable to a corollary discharge acting on these visual neurons (Richmond and Wurtz, 1977). The source of this corollary may be a direct projection from the saccaderelated neurons of the SC intermediate layers (Lee et al., 2007). We found saccadic suppression in identified PI neurons, and, as in the SC, this suppression occurs at a time appropriate to act on the visual input that results from the sweep of the eye across the visual field during saccades. Saccadic suppression has also now been seen in MT (Thiele et al., 2002; Ibbotson et al., 2007; Bremmer et al., 2009) and is more robust than in earlier visual areas such as LGN and V1 (Wurtz, 2008). This points toward the PI pathway as a promising source of the MT suppression. In this scenario, MT activity would by shaped by inputs both from preceding cortical visual areas and from the SC through the pulvinar. This possibility is now testable because identification of this SC-PI path permits inactivation of the relay conveying the suppression to MT.

A second modulation in the pulvinar pathway that we expected to see was an enhanced visual response to stimuli that are saccade targets (Goldberg and Wurtz, 1972b; Wurtz and Mohler, 1976). This enhancement has been regarded as a correlate of visual spatial attention: the stimulus is identical but the visual response increases when the monkey prepares a saccade and presumably shifts attention to the stimulus. This modulation in the superficial SC might be transmitted to visual areas of cerebral cortex and contribute to the enhanced attentional responses seen there (Wurtz et al., 1980). The ascending pulvinar path from SC to MT appeared an obvious candidate to convey these signals, but we found only a few pulvinar neurons with an enhanced visual response, and at least as many showed a decrease. The scarcity of enhancement may arise partly from procedural differences from earlier studies in superficial SC, and attentional enhancement may well be more detectable under different task conditions than those used here. Nonetheless, the controls described in Results point toward a genuine reduction in the prominence of visual enhancement in PI compared with SC despite the potential limitations of the task conditions.

One possible explanation for a genuine diminished presence of visual enhancement in PI is that this SC modulation is not transmitted at all to cortex, or is transmitted directly from presaccadic neurons in the SC intermediate layers, as suggested by the attentional effects seen with stimulation of these layers (Cavanaugh and Wurtz, 2004). Another possibility is that the enhancement is transmitted from superficial SC to cortex but by a different pathway. For example, spatially selective enhancement is characteristic of a dorsal region of the lateral pulvinar, $\mathrm{Pdm}$
(Petersen et al., 1985, 1987; Wilke et al., 2010), which was not strongly sampled in our recording. This region could mediate a projection from superficial SC to the lateral intraparietal area of cortex (Clower et al., 2001), where neurons also show attentional modulation and an enhancement in a saccade task similar to the one we used (Colby et al., 1996). In general, the uncertain transmission of visual enhancement in our identified PI pathway, coupled with the clearer presence of saccadic suppression, indicates that the SC modulatory signals may take different routes to cortex.

\section{Directional selectivity for visual motion}

The pulvinar pathway from SC has generated considerable interest as an alternate route for visual signals to reach cortex, bypassing the primary geniculostriate path. The pulvinar has been of particular interest for transmitting motion signals in blindsight, wherein patients with damage to V1 retain some visual capacities (Weiskrantz et al., 1974), often including motion detection. Yet we found little directional tuning in the PI neurons in the ascending pathway from SC to MT. By contrast, PI neurons that received input from MT were far more likely to carry directional motion signals, with $83 \%$ classified as directionally selective or biased, compared with $36 \%$ in the ascending-path PI neurons with no identifiable MT input. Tuning in the ascending-path PI neurons also stands in contrast to the identified V1 inputs to MT, which are virtually all directionally tuned (Movshon and Newsome, 1996). It is possible that the directional tuning we observe in the ascending pulvinar path, although considerably less than that of the descending path, is sufficient to support motion responses in MT. This possibility, however, rests on the assumption that the directionality we observed in the ascending-path sample is not itself dependent on MT inputs that we failed to detect. The critical test is to determine whether directional tuning in the pulvinar is abolished by MT inactivation.

Overall, our findings caution against the view of the pulvinar as an obvious source of directionally tuned inputs to MT and emphasize that it is only one of several established routes to extrastriate cortex. An alternative hypothesis (Cowey, 2004; Vakalopoulos, 2005), emerging from recent anatomical and functional studies, is that the residual visual signals seen after V1 damage are provided by direct projections from LGN to extrastriate cortex, particularly to area MT (Sincich et al., 2004). This hypothesis is supported by recent imaging studies in V1-lesioned monkeys, which demonstrate that LGN inactivation reduces both residual extrastriate activation and visual detection (Schmid et al., 2010). Neurons in this LGN relay have not yet been functionally identified, so the nature of the signals they carry is unknown. The relative contributions of these LGN and PI pathways to extrastriate visual processing will also become clearer with comparison of the deficits caused by LGN and pulvinar inactivation.

\section{Conclusion}

The pulvinar comprises the largest part of the thalamus devoted to visual and cognitive processing, yet its fundamental contributions have proved difficult to delineate. We have now identified one pathway through the pulvinar and determined some of the key signals that it does and does not convey to cortex. This opens the way to investigating its contribution to cerebral cortical function: knowing the PI relay allows for its selective inactivation and examination of the resulting deficits in cortical processing and behavior. This ascending PI pathway, however, is only one of many through the pulvinar, and 
our approach is applicable to the identification of other pathways and the signals they convey.

More generally, these findings add to emerging views of thalamic function. Traditionally, the thalamus was seen simply as a gateway for external sensory information to reach the cortex. More recent observations suggest a distinction between firstorder thalamic nuclei, which act as sensory relays, and higherorder nuclei, which convey information related to internal functions (Sherman and Guillery, 2002). Higher-order nuclei may relay internal information between cortical areas (Theyel et al., 2010) or, as is the focus here, from subcortical areas to cortex. The present study, together with previous work from this laboratory, begins to provide insight about what this internal information might be. These studies identify two paths through higher-order thalamus. A path through the mediodorsal nucleus to frontal cortex (Sommer and Wurtz, 2008) conveys a corollary discharge of saccade-related activity from the intermediate SC. The PI path to parieto-occipital cortex conveys a saccadic suppression of activity from the superficial SC. Thus, both pathways can be seen as informing cortex about movement: they convey either a corollary of movement or a consequence of that corollary. Of course, we have sampled only a small number of thalamic pathways, and the experiments are biased to reveal a relationship to movement. Nevertheless, a viable starting hypothesis is that a substantial fraction of the information ascending in these higher-order nuclei is related to a corollary discharge of movement. Whereas firstorder thalamic nuclei provide cortex with mostly sensory information from the external world, the higher-order thalamic nuclei may provide internal information concerned with movement generation, an essential signal for active perceptual processing in cortex.

\section{References}

Allman J, Miezin F, McGuinness E (1985) Direction- and velocity-specific responses from beyond the classical receptive field in the middle temporal visual area (MT). Perception 14:105-126.

Berman RA, Wurtz RH (2010) Functional identification of a pulvinar path from superior colliculus to cortical area MT. J Neurosci 30:6342-6354.

Bremmer F, Kubischik M, Hoffmann KP, Krekelberg B (2009) Neural dynamics of saccadic suppression. J Neurosci 29:12374-12383.

Britten KH, Shadlen MN, Newsome WT, Movshon JA (1992) The analysis of visual motion: a comparison of neuronal and psychophysical performance. J Neurosci 12:4745-4765.

Cavanaugh J, Wurtz RH (2004) Subcortical modulation of attention counters change blindness. J Neurosci 24:11236-11243.

Clower DM, West RA, Lynch JC, Strick PL (2001) The inferior parietal lobule is the target of output from the superior colliculus, hippocampus, and cerebellum. J Neurosci 21:6283-6291.

Colby CL, Duhamel JR, Goldberg ME (1996) Visual, presaccadic, and cognitive activation of single neurons in monkey lateral intraparietal area. J Neurophysiol 76:2841-2852.

Cowey A (2004) The 30th Sir Frederick Bartlett Lecture. Fact, artefact, and myth about blindsight. Q J Exp Psychol A 57:577-609.

Cusick CG, Scripter JL, Darensbourg JG, Weber JT (1993) Chemoarchitectonic subdivisions of the visual pulvinar in monkeys and their connectional relations with the middle temporal and rostral dorsolateral visual areas, MT and DLr. J Comp Neurol 336:1-30.

Girard P, Salin PA, Bullier J (1992) Response selectivity of neurons in area MT of the macaque monkey during reversible inactivation of area V1. J Neurophysiol 67:1437-1446.

Gizzi MS, Katz E, Schumer RA, Movshon JA (1990) Selectivity for orientation and direction of motion of single neurons in cat striate and extrastriate visual cortex. J Neurophysiol 63:1529-1543.

Goldberg ME, Wurtz RH (1972a) Activity of superior colliculus in behaving monkey: I. Visual receptive fields of single neurons. J Neurophysiol $35: 542-559$
Goldberg ME, Wurtz RH (1972b) Activity of superior colliculus in behaving monkey. II. Effect of attention on neuronal responses. J Neurophysiol 35:560-574.

Hafed ZM, Krauzlis RJ (2010) Microsaccadic suppression of visual bursts in the primate superior colliculus. J Neurosci 30:9542-9547.

Hays AV, Richmond BJ, Optican LM (1982) A UNIX-based multiple process system for real-time data acquisition and control. WESCON Conf Proc 2:1-10.

Hikosaka O, Wurtz RH (1983) Visual and oculomotor functions of monkey substantia nigra pars reticulata. III. Memory-contingent visual and saccade responses. J Neurophysiol 49:1268-1284.

Ibbotson MR, Price NS, Crowder NA, Ono S, Mustari MJ (2007) Enhanced motion sensitivity follows saccadic suppression in the superior temporal sulcus of the macaque cortex. Cereb Cortex 17:1129-1138.

Ibbotson MR, Crowder NA, Cloherty SL, Price NS, Mustari MJ (2008) Saccadic modulation of neural responses: possible roles in saccadic suppression, enhancement, and time compression. J Neurosci 28:10952-10960.

Lee PH, Sooksawate T, Yanagawa Y, Isa K, Isa T, Hall WC (2007) Identity of a pathway for saccadic suppression. Proc Natl Acad Sci U S A 104: $6824-6827$.

Lemon R (1984) Methods for neuronal recording in conscious animals. In: IBRO handbook series: methods in the neurosciences, Vol 4, pp 95-102. New York: Wiley.

Lyon DC, Nassi JJ, Callaway EM (2010) A disynaptic relay from superior colliculus to dorsal stream visual cortex in macaque monkey. Neuron 65:270-279.

Maunsell JH, Van Essen DC (1983) Functional properties of neurons in middle temporal visual area of the macaque monkey. I. Selectivity for stimulus direction, speed, and orientation. J Neurophysiol 49:1127-1147.

Movshon JA, Newsome WT (1996) Visual response properties of striate cortical neurons projecting to area MT in macaque monkeys. J Neurosci 16:7733-7741.

Petersen SE, Robinson DL, Keys W (1985) Pulvinar nuclei of the behaving rhesus monkey: visual responses and their modulations. J Neurophysiol 54:867-886.

Petersen SE, Robinson DL, Morris JD (1987) Contributions of the pulvinar to visual spatial attention. Neuropsychologia 25:97-105.

Reynolds JH, Chelazzi L (2004) Attentional modulation of visual processing. Annu Rev Neurosci 27:611-647.

Richmond BJ, Wurtz RH (1977) Visual responses during saccadic eye movement: a corollary discharge to superior colliculus. Soc Neurosci Ab$\operatorname{str} 3: 574$.

Riddoch G (1917) Dissociations of visual perceptions due to occipital injuries, with especial reference to appreciation of movement. Brain 40:15-57.

Robinson DL, Wurtz RH (1976) Use of an extraretinal signal by monkey superior colliculus neurons to distinguish real from self-induced stimulus movement. J Neurophysiol 39:852-870.

Robinson DL, Petersen SE, Keys W (1986) Saccade-related and visual activities in the pulvinar nuclei of the behaving rhesus monkey. Exp Brain Res 62:625-634.

Robinson DL, McClurkin JW, Kertzman C, Petersen SE (1991) Visual responses of pulvinar and collicular neurons during eye movements of awake, trained macaques. J Neurophysiol 66:485-496.

Rodman HR, Gross CG, Albright TD (1989) Afferent basis of visual response properties in area MT of the macaque. I. Effects of striate cortex removal. J Neurosci 9:2033-2050.

Rodman HR, Gross CG, Albright TD (1990) Afferent basis of visual response properties in area MT of the macaque. II. Effects of superior colliculus removal. J Neurosci 10:1154-1164.

Ross J, Morrone MC, Goldberg ME, Burr DC (2001) Changes in visual perception at the time of saccades. Trends Neurosci 24:113-121.

Schmid MC, Mrowka SW, Turchi J, Saunders RC, Wilke M, Peters AJ, Ye FQ, Leopold DA (2010) Blindsight depends on the lateral geniculate nucleus. Nature 466:373-377.

Sherman SM, Guillery RW (2002) The role of the thalamus in the flow of information to the cortex. Philos Trans R Soc Lond B Biol Sci 357:1695-1708.

Sincich LC, Park KF, Wohlgemuth MJ, Horton JC (2004) Bypassing V1: a direct geniculate input to area MT. Nat Neurosci 7:1123-1128.

Sommer MA, Wurtz RH (2008) Brain circuits for the internal monitoring of movements. Annu Rev Neurosci 31:317-338. 
Theyel BB, Llano DA, Sherman SM (2010) The corticothalamocortical circuit drives higher-order cortex in the mouse. Nat Neurosci 13:84-88.

Thiele A, Henning P, Kubischik M, Hoffmann KP (2002) Neural mechanisms of saccadic suppression. Science 295:2460-2462.

Treue S, Maunsell JH (1999) Effects of attention on the processing of motion in macaque middle temporal and medial superior temporal visual cortical areas. J Neurosci 19:7591-7602.

Vakalopoulos C (2005) A theory of blindsight-the anatomy of the unconscious: a proposal for the koniocellular projections and intralaminar thalamus. Med Hypotheses 65:1183-1190.

Weiskrantz L, Warrington EK, Sanders MD, Marshall J (1974) Visual capacity in the hemianopic field following a restricted occipital ablation. Brain 97:709-728.

Wilke M, Turchi J, Smith K, Mishkin M, Leopold DA (2010) Pulvinar inactivation disrupts selection of movement plans. J Neurosci 30:8650-8659.

Wurtz RH (2008) Neuronal mechanisms of visual stability. Vision Res 48:2070-2089.

Wurtz RH, Mohler CW (1976) Organization of monkey superior colliculus: enhanced visual response of superficial layer cells. J Neurophysiol 39:745-765.

Wurtz RH, Goldberg ME, Robinson DL (1980) Behavioral modulation of visual responses in the monkey: stimulus selection for attention and movement. In: Progress in psychobiology and physiological psychology (Sprague JM, Epstein AN, eds), pp 43-83. New York: Academic.

Zeki S, Ffytche DH (1998) The Riddoch syndrome: insights into the neurobiology of conscious vision. Brain 121:25-45. 